\title{
Treatment and Management in the Corona Virus Crisis: Think outside the Box While inside the Box
}

\author{
Pnina Hertz \\ Pediatric Psychologist Coordinator of Feeding Clinic-Hadassah Medical Center \\ ${ }^{\star}$ Corresponding author: Pnina Hertz, Pediatric Psychologist Coordinator of Feeding Clinic-Hadassah Medical Center; Email: PninaH2@hadassah.org.il
}

Received: April 20, 2020; Accepted: April 25, 2020; Published: April 27, 2020

\section{Perspective}

I wrote this position paper as someone who wears several hats, each one separately, and primarily, one hat on top of the other. My many personal, family-community, and professional roles overlap and enhance each other. In this period of crisis and challenge, of many difficulties and struggles, new knowledge and new experiences accumulate. In this text, I have chosen to share my personal message, coming from weeks of quarantine, and my discovery of administrative and professional modes of action that were new to me.

In ordinary times, which characteristically have greater degrees of freedom, we often encounter an avoidance approach tochange. The necessary experimentation with unfamiliar modalities/ techniques may even threaten our routine. However, in the face of another reality, there is an opportunity to grow and expand, precisely from a narrow and restricted physical boundary. I found myself in this time in therapeutic and administrative work at the Feeding Clinic, charting new ways andunfamiliar tracks. The deliberations and questionsand observations following my own process are presented below.

\section{From protocol to practice}

Protocol is formulated in routine times, shaped by habits and consistency and based on personal and collective knowledge and experience. These are accompanied by a familiar reality, which at times is nottaken into account. The relevance of accepted practicesdiminishes in times of crisis. Work protocols that have served us well until now require renewal and change, according to the given needs.

We naturally tend to prefer familiar treatment plans and methods, thinking that they will achieve optimal results. However, reliance on conventional approaches can lead to missed therapeutic and administrative opportunities that are appropriate for these periods of crisis. As we become more creative and liberated from therapeutic conventions, we can achieve a broad and refreshing newreality-based therapeutic practice. For example, telephone or e-mailsduring the corona crisis can provide a reframing of the situation as well as a calmresponse and guidance on the challenge at hand.

\section{Online treatment and management}

The interpersonal encounter is a basic component of therapeutic work in the field of early childhood mental health. The session includes eye contact in which the therapist observes emotional states and the quality of the interaction, based on mimicry and body language. The encounter sometimes invites physical contact.Treatmentina period of quarantine eliminates some elements of nonverbal communication but may well sharpen other therapeutic skills.

The richness and diversity of online treatment depends on the technological means and skills of both the therapist andthe family in treatment. The quality of the therapy is also affected by the economic level and accessible material, which is expensive. A flexible and open approachmakes remote communication with diverse populations possible and is combined withsensitivityto the populations' unique character and needs.

The advantages of remote support are both technical, such as a high level of accessibility, and qualitative. For example, in a phone conversation with parents, sensitive and attentive listening can compensate for the lack of eye contact whileways to introduce new foods to a picky eater are presented.

\section{Intake as a diagnostic and therapeutic tool}

The face-to-face intake is currently replaced by telephone intake. Many new applicants experiencea high level of distress.In many cases, it is my professionalresponsibility to refer callers to emergency medical centers after consulting with the physician in the community.

Telephone intake for non-urgent callers is usually necessaryfor parents waiting for their children's acceptance tothe feeding center. The intake is primarily intended to address their feelings of anger, frustration and disappointment. These intense emotions are not necessarily related to the child's medical condition, and often reflect the parents' cognitive and emotional state and need for an attentive ear, with or without eye contact.

\section{Anxiety vs. Distress}

Parents turn to a feeding center with possible distress in the background. How threatening or disruptive the situation is will affect the situation, as well as the parental resources that are mobilized. Currently, as the level of collective anxiety rises, the internal and private threat is sometimes temporarily reduced. Accordingly, and also because of the life-threatening possibility of increased exposure to Covid-19 virusin the medical center, parents refrain from contacting 
us unless there isimmediate danger.As therapeutic alternatives are developed; we bolster the parents' confidence who willthen appreciate conventional therapy when it becomes possible.

\section{Creativity and Productivity}

While curiosity is the primary driving force when seeking therapeutic solutions, creativity is the fuel for finding the therapeutic path. There are different examples. For instance, a toddler was initially described on the phone, as refusing to eat. He achieved independent eating and avaried dietafter one therapy (phone) call, which came after a consultation and preliminary information from the educational and rehabilitation staff of the daycare center he attended prior to quarantine with his family.

Thinking outside the box, while being physically inside the box of my home, and physically distant from the patient and his family, led me to enlist family members as modelsfor social eating. The patient's achievement delighted all those who saw themselves, and rightly so, as partners in his success.

\section{Multidisciplinary team}

The uniqueness of our feeding center is the skilled, sensitive, multidisciplinary response that can be individualized and familyoriented under one umbrella. Our staff support and complement each other so that the whole is worth more than the sum ofits parts. At this time, this integrative work continues, in shared thinking and discussion and supportive, encouraging conversations, with the hope of resuming non-virtual work sessions, as the danger passes.

Unpaid leave, layoffs and a reduced workforce at the feeding clinic as in other medical and treatment centers, arevague, unresolved, but solvable. I leave issues to the experts in economics, with the challenge of translating unique therapeutic productivity into the materialworld.

“Talk. Share. Work on you emotionally and psychologically, now is the time." Dr. Guy Winch believes that the real threat during the Corona virus's active period is mental. In an article in the Haaretzsupplement (Ayelet Shani, April 10, 2020), he emphasizes the importance of strengthening our coping tools and recommends that the mental health community not give in to helplessness oravoidance.

The routine of our lives can bring calm and stability, based on familiar and fixed events, their high predictability, clarity and logic. When I realized that the Corona virus had entered my personal and professional life, and might rob me of a stable routine, I understood theneed for a response anchored in the new reality.

This understanding led to a therapeutic thought process using methods nottypically included in early childhood mental health. The new reality has also sharpened my understanding of unique ways of managing and maintaining workrelationshipsin my work as coordinator of a multidisciplinary therapy center.

The work methods described above, adapted to extraordinary disruption, are also important for a promptresponse to non-urgent inquiries. Other cases are currently referred to emergency medical units, according to need and the recommendation of community doctors.

I warmly embrace the recommendation of psychologist Dr. Guy Winch. I do not succumb to helplessness. I invite in energized thinking and shared action in the current situation and believe that new insights are beginning to emerge. 